\title{
Diagnostic value of selected markers and apoptotic pathways for pancreatic cancer
}

\author{
ROBERT SŁOTWIŃSKII,2, SYLWIA MAEGORZATA SŁOTWINSSKA ${ }^{3}$ \\ ${ }^{1}$ Department of Surgical Research and Transplantology, Mossakowski Medical Research Centre, Polish Academy of Sciences, Poland \\ ${ }^{2}$ Department of Immunology, Biochemistry and Nutrition, Medical University of Warsaw, Poland \\ ${ }^{3}$ Department of Conservative Dentistry, Medical University of Warsaw, Poland
}

\begin{abstract}
Pancreatic cancer occupies the fourth place as a cause of death from cancer, and the mortality rate is similar to the number of newly detected cases. Due to the late diagnosis, only 5-6\% of patients with pancreatic cancer survive for five years. Given that early diagnosis is critical for improving patients' survival rates, there is an urgent need for the discovery and validation of new biomarkers with sufficient sensitivity and specificity to help diagnose pancreatic cancer early. Detection of serum tumor markers (CA19-9, CEA, CA125 and CA242) is conducive to the early diagnosis of pancreatic cancer. The combination of miR-16, miR-196a and CA19-9 plasma level was more effective, especially in early tumor screening. Furthermore, recent studies reported that mainly miR-21, miR-155 and miR-196 were dysregulated in IPMN (intraductal papillary mucinous neoplasms) and PanIN (pancreatic intraepithelial neoplasia) lesions, suggesting their usefulness as early biomarkers of these diseases. The reduced rate of apoptosis plays a crucial role in carcinogenesis, and it is one of the most important characteristics acquired by pancreatic cancer cells, which protects them from attack by the immune system and reduces the effectiveness of pharmacological treatment. This review summarizes the data concerning the clinical utility of selected biomarkers in pancreatic cancer patients. The review mainly focuses on the genetic aspects of signaling pathway disorders associated with apoptosis in the pathogenesis and diagnosis of pancreatic cancer.
\end{abstract}

Key words: pancreatic cancer, tumor markers, cell apoptosis, signaling pathways.

(Cent Eur J Immunol 2016; 41 (4): 392-403)

\section{Introduction}

Pancreatic cancer in the early stages of the disease does not give clinical symptoms, making it difficult for early diagnosis that would give hope for a successful therapy. Although there is progress in the knowledge on the pathogenesis of pancreatic cancer, only surgery performed in the early stages of the disease can significantly increase the survival. Unfortunately, due to the late diagnosis, only 5-6\% of patients with pancreatic cancer survive five years [1-3]. At the time of diagnosis of pancreatic cancer, the tumor is unresectable in $80 \%$ of patients and there are already distant metastases. The survival time of patients diagnosed with unresectable pancreatic tumor is on average 6 months and may increase only slightly (to 11 months) after the application of a complex chemotherapy [4].

One way to improve the results of the early diagnosis of pancreatic cancer and a better understanding of still unknown pathogenesis was to isolate groups particularly susceptible to the increased incidence of the disease. Meta-analyses of epidemiological studies have identified many risk factors conducive to pancreatic cancer. These are mainly smoking, older age, male gender, black race, diabetes, obesity, a diet high in fat and meat and low in vegetables and folic acid, blood type other than 0, exposure to certain chemical compounds (including nickel, $\mathrm{N}$-nitroso compounds) as well as infectious (infection with Helicobacter pylori, hepatitis C) and periodontal diseases [5]. The factors that particularly increase the risk of pancreatic cancer are chronic pancreatitis, smoking and diabetes. The risk of pancreatic cancer is significantly elevated in subjects with chronic pancreatitis and appears to be independent of sex, country, and type of pancreatitis [6-8]. The cumulative risk of pancreatic cancer in subjects who were followed for at least 2 years increased steadily, and 10 and 20 years after the diagnosis of pancreatitis it was $1.8 \%$ (95\% confidence interval [CI]: 1.0-2.6\%) and 4.0\% (95\% CI: $2.0-5.9 \%$ ), respectively [6]. The risk of developing pancreatic cancer appears to be highest in rare types of pancreatitis with an early onset, such as hereditary pancreatitis (a 53-fold higher risk for developing pancreatic cancer) and tropical pancreatitis $[9,10]$. Smoking is the

Correspondence: Robert Słotwiński, Department of Immunology, Biochemistry and Nutrition, Medical University of Warsaw, Oczki 3, 02-007 Warsaw, Poland, e-mail: robert_slotwinski@yahoo.com Submitted: 9.09.2016; Accepted: 26.09.2016 
most established risk factor for pancreatic cancer. Smoking increases the risk of pancreatic cancer almost two-fold and it is estimated that it is responsible for approximately $20-25 \%$ of pancreatic tumors, and smoker tumors carry significantly more mutations [11]. Smokers have a 2.5 to $3.6 \%$ higher risk of pancreatic cancer, as compared with nonsmokers; the risk increases with greater tobacco use and longer exposure to smoke [12]. When compared with never smokers, current smokers had a significantly elevated risk (odds ratio [OR] 1/4 1.77, 95\% CI: 1.38-2.26). The risk increased significantly with greater intensity $(\geq 30$ cigarettes/day: OR 1/4 1.75, 95\% CI: 1.27-2.42), duration ( $\geq 50$ years: OR $1 / 42.13$, 95\% CI: $1.25-3.62)$ and cumulative smoking dose ( $\geq 40$ pack-years: OR 1/4 1.78, 95\% CI: 1.35-2.34) [13]. The odds ratio of pancreatic cancer in people with type 2 diabetes is 1.8 , and is the highest in a 2-year period from the time of diagnosis and then decreases. It is estimated that approximately $1 \%$ of patients with type 2 diabetes (age 50 years or older) will develop pancreatic cancer within three years from diagnosis [14]. New-onset diabetes is associated with a 4- to 7-fold increase in risk and 1-2\% of patients with recent-onset diabetes will develop pancreatic cancer within 3 years [15]. Meta-analysis (35 cohort studies) revealed a 40-100\% increase in the risk of pancreatic cancer in diabetes mellitus [16]. Type 2 and type 1 diabetes mellitus increased the risk of pancreatic cancer with a latency period of more than 5 years. It was also reported that type 3 diabetes mellitus was an effect, and therefore a harbinger, of pancreatic cancer in at least $30 \%$ of patients [17]. Other authors believe that more studies are necessary in order to definitively identify diabetes mellitus as a risk factor for pancreatic cancer, considering the fact that approximately 10 years are needed to diagnose symptomatic pancreatic cancer [18].

At the increased risk of pancreatic cancer are also patients with a family history of the disease [19] and genetic diseases, i.e., Peutz-Jeghers syndrome (germline mutation in STK1) [20], Lynch syndrome (hereditary colorectal cancer unrelated to polyposis, caused by, i.a., microsatellite DNA instability) [21], hereditary pancreatitis (mutation in the PRSS1 gene encoding cationic trypsinogen) $[9,22]$ and hereditary breast and ovarian cancer syndrome (BRCA1 and BRCA2 gene mutations) [23, 24]. Genetic disorders observed in pancreatic cancer are often associated with KRAS oncogene activation (mutationally activated KRAS is present in $>90 \%$ of pancreatic ductal adenocarcinoma [PDAC] and represent the earliest genetic alteration), increased expression of ERBB2, inactivation of the cyclin-dependent kinase inhibitor $2 \mathrm{~A}$, p53 inactivation in about $75 \%$ of PDAC and inactivation of $C D K N 2 A$, BRCA2, SMAD4/DPC4 in up to 55\% of PDAC as well as changes in the expression of microRNAs and shortening of telomeres resulting in chromosomal instability [25-32]. Immunohistochemically detected expression of 3 major genes (CDKN2A/p16, TP53, and SMAD4/DPC4) strongly predicted the survival in patients with resectable pancreatic cancer [33] and Smad4 was correlated with lymph node metastasis and overall survival [34]. In addition, the search for non-invasive methods have shown that the salivary biomarkers possess discriminatory power for the detection of resectable pancreatic cancer with high specificity and sensitivity [35]. In saliva supernatant, four mRNA biomarkers (KRAS, MBD3L2, ACRV1, and DPM1) could differentiate pancreatic cancer patients from non-cancer subjects (chronic pancreatitis and healthy control), yielding ROC-plot AUC value of 0.971 with $90.0 \%$ sensitivity and $95.0 \%$ specificity. Furthermore, in addition to these frequently altered genes, various other genes were shown to be mutated at relatively low frequencies in pancreatic cancer (e.g., ARID1A, ARID2, MLL3, EPC1 and ATM genes related to chromatin remodeling, DNA damage repair, and genes associated with the axon guidance pathway, including $R O B O 1 / 2$ and SLIT2) [32, 36, 37]. The results of genetic studies suggest the need to personalize the therapy of pancreatic cancer. Our review is aimed at exploring the current knowledge about the diagnostic value of selected markers and apoptotic pathways for pancreatic cancer.

\section{Selected markers for early detection of pancreatic cancer}

Despite imaging modalities and histopathological and immunophenotypical evaluations of precursor lesions, only the introduction of research focused on the discovery of reliable biomarkers provided fundamental contributions to the early detection of PDAC. Recent studies showed that after tumor initiation, it could take even about 10 years for pancreatic cancer cells to acquire metastatic capacity to spread to distant organs $[38,39]$. Given that early diagnosis is critical for improving patients' survival rates, there is an urgent need for discovery and validation of new biomarkers with sufficient sensitivity and specificity to help diagnose pancreatic cancer early. Simple to assay serum-based biomarkers remain an ideal non-invasive method to detect PDAC in its early stages. Unfortunate$1 y$, at present there is no marker with sufficient diagnostic sensitivity and specificity to identify early cancer patients.

Serum carbohydrate antigen (CA19-9) is the most common conventional tumor marker analyzed in pancreatic cancer patients. Serum CA19-9 levels can provide important information with regards to prognosis, overall survival, and response to chemotherapy, and can predict post-operative recurrence, nevertheless, non-specific expression in several benign and malignant diseases limit the universal applicability of serum CA19-9 levels in pancreatic cancer management [40]. The median sensitivity of CA19-9 for diagnosis of pancreatic cancer was estimated at 79 (70-90\%) and median specificity at 82 (68-91\%). CA19-9 elevation in non-malignant jaundice resulted in a decrease in specificity [41]. The median preoperative CA 
19-9 value for patients who underwent resection was 131 $\mathrm{U} / \mathrm{ml}$ vs. $379 \mathrm{U} / \mathrm{ml}$ for patients with unresectable disease; CA19-9 values greater than $130 \mathrm{U} / \mathrm{ml}$ remained a predictor of tumor unresectability in a multivariate regression analysis [42]. Another study also confirmed that the preoperative serum levels of CA19-9 and CEA can be helpful in the prediction of resectability ( $\mathrm{R} 0$ resection) in patients with pancreatic adenocarcinoma [43]. The results of these studies confirm the link between tumor stage and the level of CA19-9. In contrast to previous studies, some recent data suggested the use of CA19-9 as a potential marker in the early detection of PDAC.

Meta-analysis performed in 3,497 participants showed that CA242 and CA19-9 had better efficacy in the diagnosis of pancreatic cancer than CEA. Furthermore, a parallel combination test of CA19-9 + CA242 might have a better diagnostic value than individual CA242 or CA19-9 tests [44]. Another recent study found that the detection of serum tumor markers (CA19.9, CEA, CA125 and CA242) is conducive to the early diagnosis of pancreatic cancer and the combined detection of tumor markers helps to improve diagnostic efficiency [45]. Moreover, CA19-9 is an independent prognostic factor for patients with pancreatic cancer. A retrospective review of an institutional EUS Pancreas Registry containing 2,083 patients showed that elevated CA19-9 indicated a greater likelihood of PDAC diagnosis relative to benign pancreatic pathology, and higher levels of CA19-9 correlated with a worse PDAC stage [46]. These authors concluded that patients with normal CA19-9 PDAC might represent a unique subclass of patients, presenting with atypical clinical features, and possibly more advanced disease stage at the time of diagnosis. Referring to the results of other study, CA19-9 and CA125 exhibited encouraging sensitivity for detecting pre-clinical pancreatic cancer, and both markers can be used as prognostic tools [47]. According to these authors, serum CA19-9 was significantly elevated up to 2 years prior to diagnosis with pancreatic cancer. The latter work challenges the prevailing view that CA19-9 is up-regulated late in the course of pancreatic cancer development.

Promising studies aimed at increasing the usefulness of CA19-9 in the early diagnosis of pancreatic cancer are based on the parallel determination of the expression of microRNA panels [48-50]. The combination of miR-16, miR-196a and CA19-9 was more effective for pancreatic cancer diagnosis, especially in early tumor screening (85.2\% in Stage I) [48]. Other studies have shown that miRNAs undergo aberrant expression in PanIN (pancreatic intraepithelial neoplasia) lesions and are likely to be important in the development of PDAC [51]. For example, microRNAs such as miR-196b, expression of which is limited to PanIN-3 lesions or pancreatic cancers, could be useful as diagnostic markers. A recent review explored the current knowledge of miRNA sampling and reported that mainly miR-21, miR-155 and miR-196 were dysregu- lated in IPMN (intraductal papillary mucinous neoplasms) and PanIN lesions, suggesting their usefulness as early biomarkers of this disease [52]. However, first of all, one need to answer the fundamental question: to what extent will the detection of CA19-9, microRNAs and other blood biomarkers really improve the early diagnosis and survival of patients with pancreatic cancer?

Another interesting direction of research aimed at improving the early diagnosis of PDAC was a parallel determination of various protein panels associated with immune response, for example, cytokines, chemokines, adhesion molecules, proteins involved in extracellular matrix degradation and lipoproteins, most often in combination with CA19-9 [53-58]. Some of these markers corresponded to the interplay between proteases (e.g., MMPs) of tumor-infiltrating neutrophils and pancreatic tumor cells. For example, the measurement of serum MMP-9 might be clinically useful for PDAC diagnosis, and proteome analysis detected elevated levels of MMP-9, DJ-1 and A1BG proteins in pancreatic juice, suggesting their further utility in PDAC diagnosis and screening [59]. Earlier studies have shown that matrix-degrading proteases (e.g., MMP-8, MMP-9 and MMP-17 metalloproteinases) and neutrophil elastase could modulate the composition of the extracellular matrix and facilitate metastasis [60]. According to another study, the imbalance of infiltrating immune cells might result in an inadequate immunologic reaction to cancer cells and the ratio of elevated neutrophils and decreased lymphocytes (NLR) could be used as a marker to assess the systemic inflammatory response and outcome [61]. In histological evaluations of pancreatic tumors, tumor-infiltrating neutrophils were preferentially associated with mucinous cystic neoplasms (MCN) and IPMN [62]. In addition, patients with pancreatic carcinoma and elevated levels of mucins, and S100A8 or S100A9 (calprotectin) inflammatory proteins in the ductal fluid, collected at the time of tumor surgical resection, were found to have significantly worse prognosis [63]. Interestingly, the intensity level of a zinc transporter, ZIP4, in endoscopic ultrasound-guided fine needle aspiration (EUS-FNA) samples was significantly associated with tumor differentiation and patient survival [64]. These results indicate that EUS-FNA is capable of non-operative detection of ZIP4, thus offering the potential for direct pre-operative detection and targeted therapy of PDAC. Acute phase proteins - neutrophil gelatinase-associated lipocalin (NGAL) and transforming growth factor- $\beta$ (TGF- $\beta$ ) family member macrophage inhibitory cytokine-1 (MIC-1) - are proposed as the best potential biomarkers specifically elevated in the blood of pancreatic cancer patients [65]. MIC-1 in combination with CA19-9 improved the diagnostic accuracy of differentiating pancreatic cancer from chronic pancreatitis and healthy individuals. As previously demonstrated for many tumor pancreatic cancer markers, MIC-1 also showed only a modest ability to distinguish between pancreatic cancer and pancreatitis and 
had a limited value in the early diagnosis of PDAC [66]. A small group of patients with pancreatic cancer and preexisting type 2 diabetes demonstrated significant elevation in the serum levels of retinol binding protein 4 (RBP-4), NGAL (neutrophil gelatinase B-associated lipocalin) and insulin-like growth factor 1 (IGF-1) together with a significant reduction in the level of insulin-like growth factor I binding protein 3 (IGFBP-3); receiver operating characteristic curve analysis revealed that theses markers could distinguish pancreatic cancer from non-pancreatic cancer cases in this study [67]. Furthermore, IGF-1R as well as IGF-1 and IGF-2 were found to be up-regulated in pancreatic cancer [68]. Moreover, serum HSP70 levels were significantly increased in patients with pancreatic cancer and could be useful as an additional biomarker for the detection of pancreatic cancer [69]. This increase in HSP70 leads to down-modulation of apoptosis and survival of cancer cells through the PI3K/AKT pathway. In pancreatic cancer, PI3K signaling pathways also regulate cellular growth, metabolism and motility. Recently, apolipoprotein AII (apoAII) isoforms and apoAII-ATQ/AT ( $C$-terminal truncations of the apoAII homo-dimer) were found to decline significantly in pancreatic cancer and might serve as plasma biomarkers for the early detection of this disease [57]. ApoAII-ATQ/AT distinguished the early stages of pancreatic cancer from healthy controls and additionally identified patients at high risk for pancreatic malignancy. In addition, the AUC values for the detection of early stage of pancreatic cancer were higher than those of CA19-9. A recent study conducted in a small group of patients pointed to a new biomarker for early detection of pancreatic cancer, i.e., glypican-1 (GPC1), which was also present on cancer-cell-derived exosomes [70]. Serum levels of this biomarker discriminated between healthy subjects and patients with a benign pancreatic disease from patients with early- and late-stage pancreatic cancer and correlated with the survival of pre- and post-surgical patients. Perhaps this biomarker will be more suitable than the previous ones, which also appeared to be very promising.

The explosion in the pancreatic cancer biomarker field enhanced the development of antibody microarrays for molecular profiling, provided insights into the nature of serum-protein alterations in pancreatic cancer patients, and showed the potential of combined measurements to improve sample classification accuracy. Recently published studies allowed identification of protein signatures associated with PDAC, displaying sensitivities and specificities in the range of $91-100 \%$ [71]. The results of the analysis suggested that $\leq 10$ protein markers were sufficient for highly accurate discrimination of PDAC. Furthermore, recombinant antibody microarrays targeting immunoregulatory and cancer-associated antigens could identify serum protein markers associated with different tumor locations in the pancreas, although this observation and its clinical implications need to be further corroborated. For the discrimination of PDAC patients from patients with benign disease, a panel of IP-10, IL-6, PDGF plus CA19-9 offered improved diagnostic performance than CA19-9 alone, and a panel of IL-8, CA19-9, IL-6 and IP-10 provided better diagnostic results in the discrimination of PDAC from chronic pancreatitis compared to CA19-9 alone [72]. Some of the key molecules associated with the immune response, including $\mathrm{C} 1$ esterase inhibitor, C3, C5, CD40, CD40 ligand, factor B, GLP-1, IFN- $\gamma$, IgM, IL-10, IL-11, IL-12, IL-13, IL-16, IL-18, IL-1-ra, IL-1 $\alpha$, IL-3, IL-5, IL-6, IL-7, and IL- 8 as well as integrin- $\alpha-11$, procathepsin $\mathrm{W}$, sialyl Lewis $x$, TGF- $\beta 1$, TNF- $\alpha$, and VEGF were shown to be differentially overexpressed in pancreatic cancer [73]. Another study showed a weak association between the level of sTNF-R2 and the development of pancreatic cancer, however, a strong correlation was observed between sTNF-R2 and diabetes as well as higher BMI [74]. Investigating inflammatory plasma markers and pancreatic cancer risk revealed that prediagnostic levels of circulating CRP, IL- 6 and TNF- $\alpha$ R2 were not associated with the risk of pancreatic cancer, suggesting that systemic inflammation, as measured by circulating inflammatory factors, was unlikely to play a major role in the development of pancreatic cancer [75]. These findings support a potential role for cytokines, chemokines and other proteins in the discrimination of PDAC from patients with benign pancreatic diseases. Considering the critical role of inflammatory proteins in the early diagnosis of pancreatic cancer, research should be focused on the identification of a panel of critical proteins using samples from early-stage pancreatic cancer patients.

The section above presented a brief overview of selected methods that facilitate early diagnosis of pancreatic cancer with particular emphasis on selected biomarkers. It clearly indicates the need to broaden the knowledge of the mechanisms that are important in the pathogenesis of pancreatic cancer. Furthermore, it is believed that in patients with PDAC more attention should be paid to the network of apoptotic signaling pathways disorders.

\section{Disorders of apoptosis}

The contribution of apoptosis to carcinogenesis of PDAC is not well defined. Genetic regulation of the signaling pathway associated with impaired apoptosis of pancreatic cancer cells is still the subject of intensive research. The activation of oncogenes and disorders in apoptosis have become the primary factors in tumorigenesis [76]. The identification of key apoptosis-related genes prior to the onset of disease will greatly help in its prevention and treatment. Cancer cells become resistant to apoptosis, as a result of genetic disturbances and impaired expression of regulatory proteins [77]. The reduced rate of apoptosis plays a crucial role in carcinogenesis, and it is one of the 
most important characteristics acquired by pancreatic cancer cells, which, among others, protects them from attack by the immune system and reduces the effectiveness of pharmacological treatment (e.g., chemotherapy) $[78,79]$. However, other studies indicated that the resistance to apoptosis should not be considered a hallmark of cancer [80]. Overexpression of Bcl-2, an antiapoptotic protein, is associated with a better survival of cancer patients. Conversely, Bax, CD95, Caspase-3 and other apoptosis-inducing proteins have been found to promote carcinogenesis.

The mechanisms of apoptosis avoidance mainly involve balance disorders between pro-apoptotic and anti-apoptotic proteins, reduced caspase function and impaired death receptor signaling [81]. Most of the experimental studies were performed on pancreatic cancer cell lines. These studies showed that the majority of pancreatic adenocarcinoma cell lines were resistant to CD95 and TRAIL-mediated apoptosis (TNF-related apoptosis-inducing ligand), despite expressing the corresponding death receptors on the cell surface [82]. The expression of Bcl-XL correlated with sensitivity to apoptosis induced by TRAIL or anti-CD95, and Bcl-XL could protect pancreatic cancer cells from CD95- and TRAIL-mediated apoptosis [83]. It was suggested that the lack of apoptosis in PanIN-1 and PanIN-2 lesions associated with pancreatic ductal adenocarcinoma was not dependent on the K-ras status and the absence of Bcl-2 expression in combination with a very low p53 immunoreactivity [84]. Other studies showed that the early overexpression of the inhibitor of apoptosis protein family (IAP, cIAP2 and Survivin) during carcinogenesis of PDAC in PanIN1 lesions as well as the anti-apoptotic IAP function was dependent on the inhibition of caspase activity $[85,86]$. Abnormal expression of the IAP family in pancreatic cancer cells was responsible for resistance to chemotherapy and down-regulation of cIAP2-induced sensitivity towards cisplatin, doxorubicin or paclitaxel [87]. Overexpression of cIAP2 was an early event in the progression of pancreatic cancer demonstrated in low- and high-grade PanIN lesions of PDAC and survival analysis revealed a shorter survival time in patients with cIAP1/ cIAP2-positive tumors [88]. These studies suggested an early contribution of the IAP protein family in tumorigenesis of PDAC.

Interestingly, other authors found that the elevated expression of FLIP (an inhibitor of caspase-8), Bcl-XL and IAP, in parallel with a down-regulation of FADD (FAS-associated death domain protein) and Bid, was common in pancreatic carcinoma cell lines resistant to apoptosis [89]. The inhibition of caspase- 8 by FAS-associated phosphatase-1 (FAP-1) and c-FLIPL, which are highly expressed in PDACs, is another important mechanism helping to evade apoptosis [90-92]. Additionally, the activation of $\mathrm{NF}-\kappa \mathrm{B}$ signaling and TRAF2 overexpression (involved in signal transduction pathways of the TNF receptor family) was also shown to contribute to the resistance towards death receptor-induced apoptosis $[82,93]$. Stat 3 and NF$\kappa \mathrm{B}$ transcription factors induced an increased anti-apoptotic Bcl-xL expression in the premalignant lesions and tumor cells [94]. These findings indicate that apoptosis resistance precedes the formation of invasive pancreatic cancer. Furthermore, pancreatic and ampullary cancer may be associated with absent Bcl-2 expression and reduced Bax, Bak and Bcl-x expression compared with normal minor ducts, while Bak and Bcl-x expression was found to be increased when compared with major ducts. Bcl-x expression correlated with survival following resection and might represent a potential prognosis marker [95]. Expression analysis of p53, Bax, and Bcl-2 using immunohistochemical staining demonstrated a statistically significant association of apoptosis with the overall survival in pancreatic cancer patients treated with surgical resection. The overexpression of Bax and Bcl-2 represents the strongest prognostic factor [96]. These data underscore the need to pay more attention to the intracellular signaling pathways that delay apoptosis of cancer cells.

One of the most interesting concepts of the pathogenesis of pancreatic cancer refers to disorders of genetic regulation of apoptosis. Recent studies have shown that genetic mutations and different signaling pathways may play an important role in the initiation and development of pancreatic cancer. It was found that pancreatic cancers contained an average of 63 genetic alterations, the majority of which were point mutations. These alterations defined a core set of 12 cellular signaling pathways and processes, all of which were genetically altered in 67 to $100 \%$ of the tumors. The main attention was paid to the signaling cascades involving KRAS signaling, Hedgehog signaling, apoptosis, control of G1/S phase transition, TGF- $\beta$ signaling and Wingless/integrase-1 (Wnt) signaling [32]. It has been shown that pancreatic cancer is an extremely heterogeneous disease that exhibits significant differences in gene expression that can differentiate three distinct tumor subtypes [97]. The major subtype of PDAC with high expression of epithelial genes was strongly dependent on KRAS signaling [98]. The ability to cause acinar-ductal metaplasia has been suggested as an important inflammation effect sensitizing the pancreas to oncogenic KRAS. Pancreatic inflammation in combination with harmful effects of environmental and genetic factors cause exacerbation of local immunosuppression by infiltrating cells, such as regulatory T cells (Treg) or myeloid-derived suppressor cells (MDSC) [99, 100]. Pancreatic cancer also showed a significant increase in macrophage infiltrations; tumor-associated macrophages, developing from peripheral blood monocytes (M2 subtype) exerted immunosuppressive effect and were associated with poor prognosis. Similarly, the M1 subtype macrophages promoted tumor growth mediated by the TNF- $\alpha$ production $[101,102]$. In addition, neutrophil infiltrations were reported in pancreat- 
ic adenocarcinomas and have been associated with the undifferentiated types of carcinoma and poor prognosis [62].

It is also known that changes in the microenvironment are connected with oncogenic KRAS signaling [103]. These data indicate that epithelial KrasG12D affects multiple cell types to drive pancreatic tumorigenesis and is essential for tumor maintenance. Since oncogenic mutations in KRAS are not sufficient to initiate carcinogenesis, secondary events, such as inflammation-induced signaling via the epidermal growth factor receptor (EGFR) and expression of the ductal Sox 9 gene are required for the accelerated formation of premalignant lesions and PDAC [104106]. Investigations in mice model revealed that EGFR signaling induced the expression of NFATc1 (nuclear factor of activated $\mathrm{T}$ cells $\mathrm{c} 1$, a family of $\mathrm{Ca} 2 \mathrm{~b} /$ calcineurin-responsive transcription factors) and Sox 9 gene transcription, leading to acinar cell transdifferentiation and initiation of pancreatic cancer [107]. As presented in the last study on transgenic mice (GEM), inflammation-induced NFATc1STAT3 transcription complex promoted pancreatic cancer initiation by KrasG12D [108]. KrasG12D mutation was reported as the most frequent genetic alteration associated with early PanIN lesions and PDAC was detected in up to $90 \%$ of patients [109]. KRAS (intracellular membrane-bound protein) activation was shown to trigger the RAF/ERK, PI3K/AKT, and NF- $\kappa \beta$ pathways causing, among others, metabolic reprogramming, persistent inflammation, changes in tumor microenvironment and resistance to apoptosis [99]. The study aimed at understanding the pathological role of the highly oncogenic KrasG12D allele in pancreatic cancer (animal model) reveled that silencing of the oncogenic Ras allele down-regulated multiple signaling pathways promoting cell proliferation, inhibiting apoptosis, breaking cell-cell contacts and regulating expression of proteases like MMP-9 [110]. Particularly interesting is the relationship between the transforming potential of oncogenic KRAS and PI3K (phosphatidylinositol 3-kinase) signaling, because mutated KRAS has been associated with up-regulation of survival signals, including the PI3K/Akt survival pathway stimulating PDAC tumorigenesis [111]. Immunohistochemical screening of human pancreatic cancer tissue demonstrated high expression of the PI3K/p110 $\gamma$ isoform (72\% of the PDAC tissue stained positive and no staining was detected in normal pancreatic ducts), which indicated a critical role of PI3K signaling in pancreatic cancer etiology [112]. It was found that the PI3K isoform activation was a marker of pancreatic cancer aggressiveness that enhanced tumor progression in the microenvironment [113]. Pharmacological or genetic blockade of PI3K/p110y suppressed inflammation, growth, and metastasis of implanted and spontaneous tumors. It is also important in apoptotic disorders that the PI3K/Akt signaling pathway is activated due to the aberrant expression of phosphatase and tensin homolog (PTEN) and target transcription factors NF- $\kappa \mathrm{B}$ and $\mathrm{c}-\mathrm{Myc}$ in pancreatic cancer cells [114]. PTEN has been one of the most studied tumor suppressor genes that exhibits anti-proliferative and proapoptotic activity [115] and is able to induce apoptosis through the AKT/PI3K pathway [116]. The loss of PTEN expression was associated with recurrence and poor prognosis in patients with PDAC and the assessment of PTEN expression might be used as a prognostic marker for patients with resected PDAC [117]. Furthermore, the increased AKT pathway activity detected in PDAC tissues and cell lines was used to induce anti-apoptotic properties of cancer cells [118, 119].

Loss of function of the $p 53$ gene by somatic inactivating mutations has been shown to occur in $50-75 \%$ of PDAC and is another important mechanisms tending to inhibit tumor cell apoptosis [120-122]. The sustained expression of the mutant TP53 allele was shown to be necessary to maintain the invasive phenotype of PDAC cells by increasing the expression of cell-autonomous platelet-derived growth factor (PDGF) receptor- $\beta$ [123]. Mutations of the TP53 tumor suppressor gene include two subtypes, a complete loss of p53 expression and overexpression of mutant p53. Interestingly, a new study revealed that the overexpression of mutant $\mathrm{p} 53$, rather than a complete loss of p53 expression, was the major genetic alteration in PDAC for patients with preoperative levels of CA19$9 \geq 1,000 \mathrm{U} / \mathrm{ml}$, which did not decrease after resection [124]. On the other hand, loss of p53 activity resulted in unleashed inflammatory responses due to the loss of p53-mediated NF- $\mathrm{KB}$ suppression and both the p53 and $\mathrm{NF}-\kappa \mathrm{B}$ pathways were commonly deregulated in cancer $[125,126]$. NF- $\kappa \mathrm{B}$ is a major factor controlling the ability of both pre-neoplastic and malignant cells to resist apoptosis-based tumor-surveillance mechanisms and behave like an oncogene [127]. Constitutive activation of NF- $\kappa \mathrm{B}$ is essential for survival and resistance to apoptosis in many tumors. In human prostate cancer lines, secreted transforming growth factor $\beta 2$ activates NF- $\kappa \mathrm{B}$, blocks apoptosis and is essential for the survival of certain tumor cells. Previous studies showed that RelA/p50NF- $\mathrm{KB}$ was constitutively activated in almost $70 \%$ of human pancreatic cancer specimens, and inhibition of NF- $\kappa \mathrm{B}$ activity by the I $\mathrm{B} \alpha$ mutant in pancreatic tumor cell lines inhibited tumorigenesis [128, 129]. In animal models of PDAC, KrasG12D mutation was the main driver of sustained constitutive IKK $\beta / N F-\kappa B$ activation through dual feedforward loops of IL-1 $\alpha /$ p62 [130]. The latest study reported a reverse correlation between NF- $\kappa \mathrm{B}$ activity in pancreatic cancer cell lines and TUSC3 (tumor suppressor candidate 3 ) expression, which was reduced in pancreatic cancer at the mRNA level [131].

A very recent work also detected low expression of RASSF6 (Ras association domain family 6) in PDAC and associated it with poor survival [132]. Only $16.7 \%$ of patients' tumor tissues were strongly positive for RASSF6 in immunostainings. These findings suggest that RASSF6 
is a valuable prognostic indicator in PDAC patients undergoing radical operations, and a decrease in RASSF6 expression may be associated with PDAC progression. RASSF6 has been identified as a new negative effector of the RAS protein that exhibits tumor suppressor activity [133]. RASSF proteins act as scaffolding agents in microtubule stability, regulate mitotic cell division, and modulate apoptosis, control cell migration and cell adhesion as well as modulate NF- $\kappa \mathrm{B}$ activity and the duration of inflammation [134]. The RASSF6 gene, down-regulated in $30-60 \%$ of a number of solid tumors, plays a role in tumourigenesis and probably acts as a regulator of apoptosis via both caspase-dependent and caspase-independent pathways [135-137]. It is essential to determine whether and how Ras triggers RASSF6-mediated apoptosis in pancreatic cancer patients.

Recently, non-coding RNAs, which are directly involved in gene expression control, have become the new direction in basic research aimed at better understanding the pathogenesis of pancreatic cancer and other diseases. It is now emerging that overexpression or down-regulation of different lncRNAs in specific types of tumors sensitize cancer cells to apoptotic stimuli. The latest findings revealed sets of intronic lncRNAs expressed in pancreatic tissues, which abundance was correlated with PDAC or metastasis [138]. The latter study identified loci harboring intronic lncRNAs (PPP3CB, MAP3K1 4 and DAPK1 loci) that were differentially expressed in PDAC metastases and were enriched in genes associated with the MAPK pathway. Aberrant overexpression of satellite repeat RNAs (HSATII) was observed in patients with PDAC, which might reflect global alterations in heterochromatin silencing and could potentially be used as biomarkers for pancreatic cancer detection [139]. HOTAIR (homeobox transcript antisense RNA), a long intervening non-coding RNA (lincRNA), was shown to be elevated in pancreatic tumors compared with non-tumor tissue, and was associated with more aggressive tumors [140]. The latter demonstrated the pro-oncogenic function of lincRNA and showed that HOTAIR knockdown in Panc1 and L3.6pL pancreatic cancer cell lines decreased cell proliferation, altered cell cycle progression and induced apoptosis. The dysregulated lncRNAs and mRNAs identified in pancreatic cancer cells might represent good candidates for future diagnostic or prognostic biomarkers and therapeutic targets [141]. Interestingly, recent work demonstrated that linc00675 overexpression positively correlated with lymph node metastasis, perineural invasion and poor survival in PDAC patients [142]. Additionally, the receiver operating characteristic curve showed that a high level of linc00675 might serve as a predictor of tumor progression. Another long non-coding RNA, HOTTIP (HOXA transcript at the distal tip), was also found to promote progression, enhance pancreatic cancer cell proliferation, survival, migration, and additionally, gemcitabine resistance by regulating HOXA13 in pancreatic cancer [143, 144]. Although the biological functions and prognostic value of lincRNAs in pancreatic cancer remain largely unexplored, these data suggest that they play a crucial role in cancer initiation, progression and metastasis $[145,146]$. As indicated by the increasing number of studies, lncRNAs are new players in the pathogenesis of pancreatic cancer, and similarly as micoRNAs, they point to a complex network of genetic links that should be better known in order to diagnose PDAC early and apply more effective pharmacotherapies.

The results of the research cited above are examples of the participation of selected signaling pathways, associated mainly with impaired apoptosis, in the pathogenesis and development of pancreatic cancer. Given the genetic heterogeneity of PDAC, when interpreting the results of these studies, one should also consider other relevant pathogenetic factors. For example, blood lymphocytes are an important factor controlling local immune disorder. The expression of Bcl-2, Bax, NF- $\mathrm{BB}$ and PARP-1 in malnourished patients with pancreatic cancer was found to be significantly lower, whereas the expression of caspases and the percentage of cells with death receptors (TNFR1/ CD120a and Fas/CD95) were significantly higher [147]. These disorders associated with cancer of the pancreas may lead to a higher lymphocyte susceptibility to accelerated apoptosis that increases immunosuppression. Earlier studies have suggested that additional genetic aberrations are needed for the progression of precursor lesions to invasive PDAC [148].

\section{Conclusions}

Failures in the treatment of pancreatic cancer are still burdened with high mortality. This fact indicates the need for verification of the state of the knowledge to find a new direction for diagnostic tests that could promptly reduce the number of patients requiring treatment in advanced disease stages. The ability to detect local changes leading to the development of pancreatic ductal adenocarcinoma (precursor lesions as PanIN, IPMNs or MCNs) in the inflamed but non-cancerous pancreatic tissue, based on expression analysis of nonspecific markers in peripheral blood, still raises concerns. Unfortunately, a large number of biomarkers discovered have not resulted in a breakthrough in the early diagnosis of pancreatic cancer. Potential biomarkers discovered in patients with pancreatic cancer reflect the misregulation of several protein-coding genes, tumor genetic changes and disorders of host immune response occurring in the course of progressive cancer. Simultaneous lack of nutrients and metabolic reprogramming, which is under the influence of both oncogenic activation and down-regulation of tumor suppressor genes, significantly hamper the search of an early marker(s) specific to pancreatic cancer. Combining conventional cancer markers with inflammatory response mediators, including the expression 
of selected microRNAs can increase their usefulness in the early diagnosis, the effectiveness of distinguishing benign from malignant lesions and monitoring and prognosis of the disease. Increasing the effectiveness of methods for early detection of disorders leading to the development of pancreatic cancer requires broadening the knowledge of the local mechanisms regulating signaling pathways associated with apoptosis. Disorders of apoptosis are of fundamental importance in the development of gastrointestinal cancers, including pancreatic cancer. The presented mechanisms of apoptosis disorders (mainly resistance to apoptosis) protect tumor cells from the immune system and chemotherapy.

The authors declare no conflict of interest.

\section{References}

1. Jemal A, Siegiel R, Xu J, Ward E (2010): Cancer Statistica 2010. CA Cancer J Clin 60: 277-300.

2. Bosetti C, Bertuccio P, Negri E, et al. (2012): Pancreatic cancer: overview of descriptive epidemiology. Mol Carcinog 51: 3-13.

3. Bosetti C, Bertuccio P, Malvezzi M, et al. (2013): Cancer mortality in Europe, 2005-2009, and an overview of trends since 1980. Ann Oncol 24: 2657-2671.

4. Conroy T, Desseigne F, Ychou M, et al. (2011): The Groupe Tumeurs Digestives of Unicancer and the PRODIGE Intergroup. FOLFIRINOX versus gemcitabine for metastatic pancreatic cancer. N Engl J Med 364: 1817-1825.

5. Klapman J, Malafa MP (2008): Early detection of pancreatic cancer: why, who, and how to screen. Cancer Control 15: 280-287.

6. Lowenfels AB, Maisonneuve P, Cavallini G, et al. (1993): Pancreatitis and the risk of pancreatic cancer. N Engl J Med 328: 1433-1437.

7. McKay CJ, Glen P, McMillan DC (2008): Chronic inflammation and pancreatic cancer. Best Pract Res Clin Gastroenterol 22: 65-73.

8. Raimondi S, Lowenfels AB, Morselli-Labate AM, et al. (2010): Pancreatic cancer in chronic pancreatitis; etiology, incidence, and early detection. Best Pract Res Clin Gastroenterol 24: 349-358.

9. Lowenfels AB, Maisonneuve P, DiMagno EP, et al. (1997): Hereditary pancreatitis and the risk of pancreatic cancer. International Hereditary Pancreatitis Study Group. J Natl Cancer Inst 89: 442-446.

10. Klein AP (2012): Genetic susceptibility to pancreatic cancer. Mol Carcinog 51: 14-24.

11. Raimondi S, Maisonneuve P, Lowenfels AB (2009): Epidemiology of pancreatic cancer: an overview. Nat Rev Gastroenterol Hepatol 6: 699-708.

12. Hassan MM, Bondy ML, Wolff RA, et al. (2007): Risk factors for pancreatic cancer: case control study. Am J Gastroenterol 102: 2696-2707.

13. Lynch SM, Vrieling A, Lubin JH, et al. (2009): Cigarette smoking and pancreatic cancer: a pooled analysis from the pancreatic cancer cohort consortium. Am J Epidemiol 170: 403-413.
14. Chari ST, Leibson CL, Rabe KG, et al. (2005): Probability of pancreatic cancer following diabetes: A population-based study. Gastroenterology 129: 504-511.

15. Pannala R, Basu A, Petersen GM, Chari ST (2009): New-onset Diabetes: A Potential Clue to the Early Diagnosis of Pancreatic Cancer. Lancet Oncol 10: 88-95.

16. Ben Q, Xu M, Ning X, et al. (2011): Diabetes mellitus and risk of pancreatic cancer: A meta-analysis of cohort studies. Eur J Cancer 47: 1928-1937.

17. Magruder JT, Elahi D, Andersen DK (2011): Diabetes and pancreatic cancer:chicken or egg? Pancreas 40: 339-351.

18. Pezzilli R, Pagano N (2013): Is diabetes mellitus a risk factor for pancreatic cancer? World J Gastroenterol 19: 4861-4866.

19. Klein AP, Brune KA, Petersen GM (2004): Prospective risk of pancreatic cancer in familial pancreatic cancer kindreds. Cancer Res 64: 2634-2638.

20. Korsse SE, Harinck F, van Lier MG, et al. (2013): Pancreatic cancer risk in Peutz-Jeghers syndrome patients: a large cohort study and implications for surveillance. J Med Genet 50: 59-64.

21. Kastrinos F, Mukherjee B, Tayob N, et al. (2009): Risk of Pancreatic Cancer in Families With Lynch Syndrome. JAMA 302: 1790-1795.

22. Weiss F (2014): Pancreatic cancer risk in hereditary pancreatitis. Front Physiol 5: 1-5.

23. van Asperen CJ, Brohet RM, Meijers-Heijboer EJ, et al. (2005): Netherlands Collaborative Group on Hereditary Breast Cancer (HEBON) Cancer risks in BRCA2 families: estimates for sites other than breast and ovary. J Med Genet 42: 711-719.

24. Thompson D, Easton DF (2002): Breast Cancer Linkage Consortium. Cancer Incidence in BRCA1 mutation carriers. J Natl Cancer Inst 94: 1358-1365.

25. Yachida S, Iacobuzio-Donahue CA (2013): Evolution and dynamics of pancreatic cancer progression. Oncogene 32: 5253-5260.

26. Shin SH, Kim SC, Hong SM, et al. (2013): Genetic alterations of K-ras, p53, c-erbB-2, and DPC4 in pancreatic ductal adenocarcinoma and their correlation with patient survival. Pancreas 42: 216-222.

27. Huggett MT, Pereira SP (2011): Diagnosing and managing pancreatic cancer. Practitioner 255: 21-33.

28. Al-Sukhni W, Borgida A, Rothenmund H, et al. (2012): Screening for pancreatic cancer in a high-risk cohort: an eight-year experience. J Gastrointest Surg 16: 771-783.

29. Kent OA, Mullendore M, Wentzel EA, et al. (2009): A resource for analysis of microRNA expression and function in pancreatic ductal adenocarcinoma cells. Cancer Biol Ther 8: 2013-2024

30. Yonezawa S, Higashi M, Yamada N, Goto M (2008): Precursor lesions of pancreatic cancer. Gut Liver 2: 137-154.

31. Schneider G, Schmid RM (2003): Genetic alterations in pancreatic carcinoma. Molecular Cancer 2: 1-7.

32. Jones $S$, Zhang X, Parsons DW, et al. (2008): Core signaling pathways in human pancreatic cancers revealed by global genomic analyses. Science 321: 1801-6.

33. Oshima M, Okano K, Muraki S, et al. (2013): Immunohistochemically detected expression of 3 major genes (CDKN2A/ p16, TP53, and SMAD4/DPC4) strongly predicts survival in patients with resectable pancreatic cancer. Ann Surg 258: 336-346. 
34. Jiang H, He C, Geng S, et al. (2012): RhoT1 and Smad4 are correlated with lymph node metastasis and overall survival in pancreatic cancer. PLoS One 7: e42234.

35. Zhang L, Farrell JJ, Zhou H, et al. (2010): Salivary Transcriptomic Biomarkers for Detection of Resectable Pancreatic Cancer Gastroenterology 138: 949-957.

36. Biankin AV, Waddell N, Kassahn KS, et al. (2012): Pancreatic cancer genomes reveal aberrations in axon guidance pathway genes. Nature 491: 399-405.

37. Waddell N, Pajic M, Patch AM, et al. (2015): Whole genomes redefine the mutational landscape of pancreatic cancer. Nature 518: 495-501.

38. Campbell PJ, Yachida S, Mudie LJ, et al. (2010): The patterns and dynamics of genomic instability in metastatic pancreatic cancer. Nature 467: 1109-1113.

39. Yachida S, Jones S, Bozic I, et al. (2010): Distant metastasis occurs late during the genetic evolution of pancreatic cancer. Nature 467: 1114-1117.

40. Ballehaninna UK, Chamberlain RS (2012): The clinical utility of serum CA 19-9 in the diagnosis, prognosis and management of pancreatic adenocarcinoma: An evidence based appraisal. J Gastrointest Oncol 3: 105-119.

41. Goonetilleke KS, Siriwardena AK (2007): Systematic review of carbohydrate antigen (CA 19-9) as a biochemical marker in the diagnosis of pancreatic cancer. Eur J Surg Oncol 33: 266-270.

42. Maithel SK, Maloney S, Winston C, et al. (2008): Preoperative CA 19-9 and the yield of staging laparoscopy in patients with radiographically resectable pancreatic adenocarcinoma. Ann Surg Oncol 15: 3512-3520.

43. Kim YC, Kim HJ, Park JH, et al. (2009): Can preoperative CA19-9 and CEA levels predict the resectability of patients with pancreatic adenocarcinoma? J Gastroenterol Hepatol 24: 1869-1875.

44. Zhang Y, Yang J, Li H, et al. (2015): Tumor markers CA199, CA242 and CEA in the diagnosis of pancreatic cancer: a meta-analysis. Int J Clin Exp Med 8: 11683-11691.

45. Gu YL, Lan Ch, Pei H, et al. (2015): Applicative value of serum CA19-9, CEA, CA125 and CA242 in diagnosis and prognosis for patients with pancreatic cancer treated by concurrent chemoradiotherapy. Asian Pac J Cancer Prev 16: 6569-6573.

46. Parikh DA, Durbin-Johnson B, Urayama S (2014): Utility of serum CA19-9 levels in the diagnosis of pancreatic ductal adenocarcinoma in an endoscopic ultrasound referral population. J Gastrointest Cancer 45: 74-79.

47. O'Brien DP, Sandanayake NS, Jenkinson C, et al. (2015): Serum CA19-9 is significantly up-regulated up to 2 years prior to diagnosis with pancreatic cancer: implications for early disease detection. Clin Cancer Res 21: 622-631.

48. Liu J, Gao J, Du Y, et al. (2012): Combination of plasma microRNAs with serum CA19-9 for early detection of pancreatic cancer. Int J Cancer 131: 683-691.

49. Schultz NA, Dehlendorff C, Jensen BV, et al. (2014): MicroRNA biomarkers in whole blood for detection of pancreatic cancer. JAMA 311: 392-404.

50. Buchsbaum DJ, Croce CM (2014): Will detection of MicroRNA biomarkers in blood improve the diagnosis and survival of patients with pancreatic cancer? JAMA 311: 363-365.

51. Yu J, Li A, Hong SM, et al. (2012): MicroRNA Alterations of Pancreatic Intraepithelial Neoplasms (PanINs) Clin Cancer Res 18: 981-992.
52. Hernandez YG, Lucas AL (2016): MicroRNA in pancreatic ductal adenocarcinoma and its precursor lesions. World J Gastrointest Oncol 8: 18-29.

53. Simeone DM, Ji B, Banerjee M, Arumugam T, et al. (2007): CEACAM1, a novel serum biomarker for pancreatic cancer. Pancreas 34: 436-443.

54. Ingvarsson J, Wingren C, Carlsson A, et al. (2008): Detection of pancreatic cancer using antibody microarray-based serum protein profiling. Proteomics 8: 2211-2219.

55. Winter M, Ting AH, Vilardell F, et al. (2008): Absence of E-Cadherin Expression Distinguishes Noncohesive from Cohesive Pancreatic Cancer. Clin Cancer Res 14: 412-418.

56. Joergensen MT, Brünner N, De Muckadell OB (2010): Comparison of circulating MMP-9, TIMP-1 and CA19-9 in the detection of pancreatic cancer. Anticancer Research 30 : 587-592.

57. Honda K, Kobayashi M, Okusaka T, et al. (2015): Plasma biomarker for detection of early stage pancreatic cancer and risk factors for pancreatic malignancy using antibodies for apolipoprotein-AII isoforms. Sci Rep 5: 15921(1-15).

58. Felix K, Gaida MM (2016): Neutrophil-derived proteases in the microenvironment of pancreatic cancer-active players in tumor progression. Int J Biol Sci 12: 302-313.

59. Tian M, Cui YZ, Song GH, et al. (2008): Proteomic analysis identifies MMP-9, DJ-1 and A1BG as overexpressed proteins in pancreatic juice from pancreatic ductal adenocarcinoma patients. BMC Cancer 8: 241-252.

60. Gregory AD, Houghton AM (2011): Tumor-associated neutrophils: new targets for cancer therapy. Cancer Res 71 : 2411-2416.

61. Yang JJ, Hu ZG, Shi WX, et al. (2015): Prognostic significance of neutrophil to lymphocyte ratio in pancreatic cancer: A meta-analysis. World J Gastroenterol 21: 2807-2815.

62. Reid MD, Basturk O, Thirabanjasak D, et al. (2011): Tumor-infiltrating neutrophils in pancreatic neoplasia. Mod Pathol 24: 1612-1619.

63. Chen KT, Kim PD, Jones KA, et al. (2014): Potential prognostic biomarkers of pancreatic cancer. Pancreas 43: 22-27.

64. Xu C, Wallace MB, Yang J, et al. (2014): ZIP4 is a novel diagnostic and prognostic marker in human pancreatic cancer: a systemic comparison between EUS-FNA and surgical specimens. Curr Mol Med 14: 309-315.

65. Kaur S, Chakraborty S, Baine MJ, et al. (2013): Potentials of plasma NGAL and MIC-1 as biomarker(s) in the diagnosis of lethal pancreatic cancer. PLoS One 8: e55171.

66. Koopmann J, Buckhaults P, Brown DA, et al. (2004): Serum macrophage inhibitory cytokine 1 as a marker of pancreatic and other periampullary cancers. Clinical Cancer Research 10: $2386-2392$

67. El-Mesallamy HO, Hamdy NM, Zaghloul AS, Sallam AM (2013): Clinical value of circulating lipocalins and insulin-like growth factor axis in pancreatic cancer diagnosis. Pancreas 42: 149-154.

68. Lopez T, Hanahan D (2002): Elevated levels of IGF-1 receptor convey invasive and metastatic capability in a mouse model of pancreatic islet tumorigenesis. Cancer Cell 1: 339353.

69. Dutta SK, Girotra M, Singla M, et al. (2012): Serum HSP70: a novel biomarker for early detection of pancreatic cancer. Pancreas 41: 530-534.

70. Melo SA, Luecke LB, Kahlert C, et al. (2015): Glypican-1 identifies cancer exosomes and detects early pancreatic cancer. Nature 523: 177-182. 
71. Gerdtsson AS, Malats N, Säll A, et al. (2015): A Multicenter Trial Defining a Serum Protein Signature Associated with Pancreatic Ductal Adenocarcinoma. Int J Proteomics 2015: 587250.

72. Shaw VE, Lane B, Jenkinson C, et al. (2014): Serum cytokine biomarker panels for discriminating pancreatic cancer from benign pancreatic disease. Mol Cancer 13: 114.

73. Wingren C, Sandström A, Segersvärd R, et al. (2012): Identification of serum biomarker signatures associated with pancreatic cancer. Cancer Res 72: 2481-2490.

74. Grote VA, Kaaks R, Nieters A, et al. (2012): Inflammation marker and risk of pancreatic cancer: a nested case-control study within the EPIC cohort. Br J Cancer 106: 1866-1874.

75. Bao Y, Giovannucci EL, Kraft P, et al. (2013): Inflammatory Plasma Markers and Pancreatic Cancer Risk: a Prospective Study of 5 U.S. Cohorts. Cancer Epidemiol Biomarkers Prev 22: 855-861.

76. Hanahan D, Weinberg RA (2000): The hallmarks of cancer. Cell 100: 57-70.

77. Hanahan D, Weinberg RA (2011): Hallmarks of Cancer: The Next Generation. Cell 144: 646-674.

78. Wong HH, Lemoine NR (2009): Pancreatic cancer: molecular pathogenesis and new therapeutic targets. Nat Rev Gastroenterol Hepatol 6: 412-422.

79. Fulda S, Galluzzi L, Kroemer G (2010): Targeting mitochondria for cancer therapy. Nat Rev Drug Discov 9: 447464.

80. Wang RA, Li ZS, Yan QG, et al. (2014): Resistance to apoptosis should not be taken as a hallmark of cancer. Chin J Cancer 33: 47-50.

81. Wong RSY (2011): Apoptosis in cancer: from pathogenesis to treatment. Journal of Experimental and Clinical Cancer Research 30: 87.

82. Trauzold A, Wermann H, Arlt A, et al. (2001): CD95 and TRAIL receptor-mediated activation of protein kinase $\mathrm{C}$ and $\mathrm{NF}-\mathrm{KB}$ contributes to apoptosis resistance in ductal pancreatic adenocarcinoma cells. Oncogene 20: 4258-4269.

83. Hinz S, Trauzold A, Boenicke L, et al. (2000): Bcl-XL protects pancreatic adenocarcinoma cells against CD95- and TRAIL-receptor-mediated apoptosis. Oncogene 19: 5477 5486.

84. Lüttges J, Neumann S, Jesenofsky R, et al. (2003): Lack of apoptosis in PanIN-1 and PanIN-2 lesions associated with pancreatic ductal adenocarcinoma is not dependent on K-ras status. Pancreas 27: e57-62.

85. Fulda S (2007): Inhibitor of apoptosis proteins as targets for anticancer therapy. Expert Rev Anticancer Ther 7: 12551264.

86. Bhanot U, Heydrich R, Moller P, Hasel C (2006): Survivin expression in pancreatic intraepithelial neoplasia (PanIN): steady increase along the developmental stages of pancreatic ductal adenocarcinoma. Am J Surg Pathol 30: 754-759.

87. Lopes RB, Gangeswaran R, McNeish IA, et al. (2007): Expression of the IAP protein family is dysregulated in pancreatic cancer cells and is important for resistance to chemotherapy. Int J Cancer 120: 2344-2352.

88. Esposito I, Kleeff J, Abiatari I, et al. (2007): Overexpression of cellular inhibitor of apoptosis protein 2 is an early event in the progression of pancreatic cancer. J Clin Pathol 60: 885-895.

89. Trauzold A, Schmiedel S, Roder C, et al. (2003): Multiple and synergistic deregulations of apoptosis-controlling genes in pancreatic carcinoma cells. Br J Cancer 89: 1714-1721.
90. Ungefroren H, Voss M, Jansen M, et al. (1998): Human pancreatic adenocarcinomas express Fas and Fas ligand yet are resistant to Fas-mediated apoptosis. Cancer Res 58: 17411749.

91. Ungefroren H, Kruse ML, Trauzold A, et al. (2001): FAP-1 in pancreatic cancer cells: functional and mechanistic studies on its inhibitory role in CD95-mediated apoptosis. J Cell Sci 114: $2735-2746$

92. Elnemr A, Ohta T, Yachie A, et al. (2001): Human pancreatic cancer cells disable function of Fas receptors at several levels in Fas signal transduction pathway. Int J Oncol 18: 311-316.

93. Trauzold A, Roder C, Sipos B, et al. (2005): CD95 and TRAF2 promote invasiveness of pancreatic cancer cells. Faseb J 19: 620-622.

94. Greten FR, Weber CK, Greten TF, et al. (2002): Stat3 and NF-kappaB activation prevents apoptosis in pancreatic carcinogenesis. Gastroenterology 123: 2052-2063.

95. Evans JD, Cornford PA, Dodson A, et al. (2001): Detailed tissue expression of bcl-2, bax, bak and bcl-x in the normal human pancreas and in chronic pancreatitis, ampullary and pancreatic ductal adenocarcinomas. Pancreatology 1: 254262.

96. Magistrelli P, Coppola R, Tonini G, et al. (2006): Apoptotic index or a combination of $\mathrm{Bax} / \mathrm{Bcl}-2$ expression correlate with survival after resection of pancreatic adenocarcinoma. J Cell Biochem 97: 98-108.

97. Cowley MJ, Chang DK, Pajic M, et al. (2013): Understanding pancreatic cancer genomes. J Hepatobiliary Pancreat Sci 20: 549-556.

98. Collisson EA, Sadanandam A, Olson P, et al. (2011): Subtypes of pancreatic ductal adenocarcinoma and their differing responses to therapy. Nat Med 17: 500-503.

99. Pylayeva-Gupta Y, Grabocka E, Bar-Sagi D (2011): RAS oncogenes: weaving a tumorigenic web. Nat Rev Cancer 11: 761-774.

100. Steele CW, Jamieson NB, Evans TR, et al. (2013): Exploiting inflammation for therapeutic gain in pancreatic cancer. Br J Cancer 108: 997-1003.

101. Kurahara H, Shinchi H, Mataki Y, et al. (2011): Significance of M2-polarized tumor-associated macrophage in pancreatic cancer. J Surg Res 167: e211-e219.

102. Baran B, Bechyne I, Siedlar M, et al. (2009): Blood monocytes stimulate migration of human pancreatic carcinoma cells in vitro: the role of tumour necrosis factor-alpha. Eur J Cell Biol 88: 743-752.

103. Collins MA, Bednar F, Zhang Y, et al. (2012): Oncogenic Kras is required for both the initiation and maintenance of pancreatic cancer in mice. J Clin Invest 122: 639-653.

104. Guerra C, Schuhmacher AJ, Cańamero M, et al. (2007): Chronic pancreatitis is essential for induction of pancreatic ductal adenocarcinoma by K-Ras oncogenes in adult mice. Cancer Cell 11: 291-302.

105. Reichert M, Rustgi AK (2011): Pancreatic ductal cells in development, regeneration, and neoplasia. J Clin Invest 121: 4572-4578.

106. Kopp JL, von Figura G, Mayes E, et al. (2012): Identification of Sox9-dependent acinar-to-ductal reprogramming as the principal mechanism for initiation of pancreatic ductal adenocarcinoma. Cancer Cell 22: 737-750.

107. Chen NM, Singh G, Koenig A, et al. (2015): NFATc1 Links EGFR Signaling to Induction of Sox9 Transcription and Ac- 
inar-Ductal Transdifferentiation in the Pancreas. Gastroenterology 148: 1024-1034.

108. Baumgart S, Chen NM, Siveke JT, et al. (2014): Inflammation-induced NFATc1-STAT3 transcription complex promotes pancreatic cancer initiation by KrasG12D. Cancer Discov 4: 688-701.

109. di Magliano MP, Logsdon CD (2013): Roles for KRAS in pancreatic tumor development and progression. Gastroenterology 144: 1220-1229.

110. Rachagani S, Senapati S, Chakraborty S, et al. (2011): Activated $\mathrm{KrasG}^{12} \mathrm{D}$ is associated with invasion and metastasis of pancreatic cancer cells through inhibition of E-cadherin. Br J Cancer 104: 1038-1048.

111. Falasca M, Selvaggi F, Buus R, et al. (2011): Targeting phosphoinositide 3-kinase pathways in pancreatic cancer - from molecular signalling to clinical trials. Anticancer Agents Med Chem 11: 455-463.

112. Edling CE, Selvaggi F, Buus R, et al. (2010): Key role of phosphoinositide 3-kinase class IB in pancreatic cancer. Clin Cancer Res 16: 4928-4937.

113. Schmid MC, Avraamides CJ, Dippold HC, et al. (2011): Receptor tyrosine kinases and TLR/IL1Rs unexpectedly activate myeloid cell PI3k $\gamma$, a single convergent point promoting tumor inflammation and progression. Cancer Cell 19: 715-727.

114. Asano T, Yao Y, Zhu J, et al. (2004): The PI 3-kinase/Akt signaling pathway is activated due to aberrant Pten expression and targets transcription factors NF-kappaB and c-Myc in pancreatic cancer cells. Oncogene 23: 8571-8580.

115. Yamada KM, Araki M (2001): Tumor suppressor PTEN: modulator of cell signaling, growth, migration and apoptosis. J Cell Sci 114: 2375-2382.

116. Weng LP, Gimm O, Kum JB, et al. (2001): Transient ectopic expression of PTEN in thyroid cancer cell lines induces cell cycle arrest and cell type-dependent cell death. Human Molecular Genetics 10: 251-258.

117. Foo WCh, Rashid A, Wang H, et al. (2013): Loss of PTEN Expression Is Associated with Recurrence and Poor Prognosis in Patients with Pancreatic Ductal Adenocarcinoma. Hum Pathol 44: 1024-1030.

118. Franke TF (2008): PI3K/Akt: getting it right matters. Oncogene 27: 6473-6488.

119. Bleeker FE, Felicioni L, Buttitta F, et al. (2008): AKT1(E17K) in human solid tumours. Oncogene 27: 56485650.

120. Vousden KH. Prives C (2009): Blinded by the Light: The Growing Complexity of p53. Cell 137: 413-431.

121. Li D, Xie K, Wolff R, Abbruzzese JL (2004): Pancreatic cancer. Lancet 363: 1049-1057.

122. Rozenblum E, Schutte M, Goggins M, et al. (1997): Tumor-suppressive Pathways in Pancreatic Carcinoma. Cancer Res 57: 1731-1734.

123. Weissmueller S, Manchado E, Saborowski M, et al. (2014): Mutant p53 drives pancreatic cancer metastasis through cell-autonomous PDGF receptor $\beta$ signaling. Cell 157: 382394.

124. Xiang J-F, Wang WQ, Liu L, et al. (2016): Mutant p53 determines pancreatic cancer poor prognosis to pancreatectomy through upregulation of cavin-1 in patients with preoperative serum CA19-9 $\geq 1,000$ U/mL. Sci Rep 6: 19222.

125. Karin M (2006): Nuclear factor-kappaB in cancer development and progression. Nature 441: 431-436.
126. Levine AJ, Oren M (2009): The first 30 years of p53: growing ever more complex. Nat Rev Cancer 9: 749-758.

127. Lu T, Burdelya LG, Swiatkowski SM, et al. (2004): Secreted transforming growth factor beta2 activates NF-kappaB, blocks apoptosis, and is essential for the survival of some tumor cells. Proc Natl Acad Sci U S A 101: 7112-7117.

128. Wang W, Abbruzzese JL, Evans DB, et al. (1999): The nuclear factor-nB RelA transcription factor is constitutively activated in human pancreatic adenocarcinoma cells. Clin Cancer Res 5: 119-127.

129. Fujioka S, Sclabas GM, Schmidt C, et al. (2003): Inhibition of constitutive NF-kappa B activity by I kappa B alpha M suppresses tumorigenesis. Oncogene 22: 1365-1370.

130. Ling J, Kang Y, Zhao R, et al. (2012): KrasG12D-induced IKK $2 / \beta / N F-\kappa B$ activation by IL- $1 \alpha$ and $p 62$ feedforward loops is required for development of pancreatic ductal adenocarcinoma. Cancer Cell 21: 105-120.

131. Fan X, Zhang X, Shen J, et al. (2016): Decreased TUSC3 Promotes Pancreatic Cancer Proliferation, Invasion and Metastasis. PLoS One 11: e0149028.

132. Ye HL, Li DD, Lin Q, et al. (2015): Low RASSF6 expression in pancreatic ductal adenocarcinoma is associated with poor survival. World J Gastroenterol 21: 6621-6630.

133. Iwasa H, Jiang X, Hata Y (2015): RASSF6; the Putative Tumor Suppressor of the RASSF Family. Cancers 7: 24152426.

134. Volodko N, Gordon M, Salla M, et al. (2014): RASSF tumor suppressor gene family: biological functions and regulation. FEBS Lett 588: 2671-2684.

135. van der Weyden L, Adams DJ (2007): The Ras-association domain family (RASSF) members and their role in human tumourigenesis. Biochim Biophys Acta 1776: 58-85.

136. Allen NP, Donninger H, Vos MD, et al. (2007): RASSF6 is a novel member of the RASSF family of tumor suppressors. Oncogene 26: 6203-6211.

137. Ikeda M, Hirabayashi S, Fujiwara N, et al. (2007): Ras-association domain family protein 6 induces apoptosis via both caspase-dependent and caspase-independent pathways. Exp Cell Res 313: 1484-1495.

138. Tahira AC, Kubrusly MS, Faria MF, et al. (2011): Long noncoding intronic RNAs are differentially expressed in primary and metastatic pancreatic cancer. Mol Cancer 10: 141.

139. Ting DT, Lipson D, Paul S, et al. (2011): Aberrant overexpression of satellite repeats in pancreatic and other epithelial cancers. Science 331: 593-596.

140. Kim K, Jutooru I, Chadalapaka G, et al. (2013): HOTAIR is a negative prognostic factor and exhibits pro-oncogenic activity in pancreatic cancer. Oncogene 32: 1616-1625.

141. Zhou M, Ye Z, Gu Y, et al. (2015): Genomic analysis of drug resistant pancreatic cancer cell line by combining long non-coding RNA and mRNA expression profling. Int J Clin Exp Pathol 8: 38-52.

142. Li DD, Fu ZQ, Lin Q, et al. (2015): Linc00675 is a novel marker of short survival and recurrence in patients with pancreatic ductal adenocarcinoma. World J Gastroenterol 21: 9348-9357.

143. Li Z, Zhao X, Zhou Y, et al. (2015): The long non-coding RNA HOTTIP promotes progression and gemcitabine resistance by regulating HOXA13 in pancreatic cancer. J Transl Med 13: 84.

144. Cheng Y, Jutooru I, Chadalapaka G, et al. (2015): The long non-coding RNA HOTTIP enhances pancreatic cancer cell 
proliferation, survival and migration. Oncotarget 6: 1084010852.

145. Gutschner T, Diederichs S (2012): The hallmarks of cancer: a long noncoding RNA point of view. RNA Biol 9: 703-719.

146. Gupta RA, Shah N, Wang KC, et al. (2010): Long non-coding RNA HOTAIR reprograms chromatin state to promote cancer metastasis. Nature 464: 1071-1076.

147. Słotwiński R, Olszewski WL, Słodkowski M, et al. (2011): Apoptosis in lymphocytes of pancreatic cancer patients: influence of preoperative enteral immunonutrition and extensive surgery. Arch Immunol Ther Exp 59: 385-397.

148. Morris JP, Wang SC, Hebrok M (2010): KRAS, Hedgehog, Wnt and the twisted developmental biology of pancreatic ductal adenocarcinoma. Nat Rev Cancer 10: 683-695. 